Maltz

Published 1999, Human Factors vol. 41, No. 1, pp.15-25

\title{
EYE MOVEMENTS OF YOUNGER AND OLDER DRIVERS
}

Masha Maltz and David Shinar ${ }^{1}$, Ben-Gurion University of the Negev, Israel

This 2-part study focuses on eye movements to explain driving-related visual performance in younger and older subjects. In the first task, subjects' eye movements were monitored as they viewed a traffic scene image with a numeric overlay, and visually located the numbers in their sequential order. The results showed that older subjects had significantly longer search episodes than younger subjects, and their visual search was characterized by more fixations and shorter saccades, although the average fixation durations remained the same. In the second task the subjects viewed pictures of traffic scenes photographed from the driver's perspective, and the task was to assume the role of the driver and regard the image accordingly. Results in the second task showed that older subjects allocated a larger percentage of their visual scan time to a small subset of areas in the image, while younger subjects scanned the images more evenly. Also, older subjects revisited the same areas and younger subjects did not. The results suggest how aging might affect the efficacy of visual information processing.

Running Title: EYE MOVEMENTS OF DRIVERS

Key Words: Eye movements, Older drivers

${ }^{1}$ Requests for reprints should be sent to David Shinar, Department of Industrial Engineering and Management, Ben Gurion University of the Negev, PO Box 653, Beer Sheva 84105, Israel 
Maltz

\section{INTRODUCTION}

There is much experimental data to support the casual observation that visual perception and information processing capabilities decline with advancing age. Because driving is a highly visual task, this deterioration has been used to account for higher accident involvement by older drivers [e.g.; Owsley et al., 1991; Staplin et al., 1987; Shinar, 1978]. Further research has focused on the correlation between vision and driving ability in older drivers [Szlyk et al., 1995(a), (b); Ball et al., 1993; Shinar and Schieber, 1991; Shinar 1977; Rackoff, 1975].

One way to better understand visually mediated attention and information processing is to analyze eye movements. There has been much research done on how eye movements relate to artificial or natural scene perception in various types of visual tasks [e.g. Rayner and Pollatsek, 1992; Whiteside, 1974], including specifically people engaged in driving tasks [Serafin, 1994; Whalen et al., 1968; McMahan, 1973; Rockwell, 1972]. Also, differences in eye movement spatial patterns and saccadic reaction times have been related to age in various studies. Whiteside [1974] compared the eye movements of older and younger adults as they viewed dot patterns. When the older group viewed the stimulus, their foveal fixations encompassed a larger area of the stimulus relative to those of the younger adult group, indicating poorer peripheral vision. Similarly, other studies have found decreased peripheral vision or poorer visual tracking skills in older people [Olincy et al., 1997; Bono et al., 1996; Kline, 1994; Kanayama et al., 1994]

Several studies have focused on more complex visual/attentional performance. For example, in an extensive series of papers, Ball and her associates [Ball et al., 1988; Ball and Owsley, 1991; Ball et al., 1993; Ball and Rebok, 1994] defined a useful field of view (UFOV) as the visual area from which information can be processed during a single fixation while a person is engaged in a foveal task. They found that the UFOV area decreases with advancing age but can be broadened with training, which may help accident-prone older drivers. They also found that UFOV was found 
Maltz

to be a better measure for accounting for crash involvement than age, visual acuity, or mental status in older drivers.

Despite the undeniable link between vision and driving, and the documented deterioration of vision with age, attempts to relate age and/or visual impairment to actual driver performance have yielded mixed results. Szlyk et al. (1995a) found that while drivers with visual impairment performed less well on driving simulators, it did not reflect real-life accident rates. Ball et al. (1993) found that inattention was a more reliable predictor of crashes than visual functions or eye health.

In a study on 237 subjects, Schieber [1989] designed the Complex Visual Reaction Time (CVRT) test to determine age-related differences in visual search time. In this test a subject is presented with a picture of a traffic scene on which 14 numbers (114) are superimposed in a random order (see Figure 1). The task is to locate sequentially as many numbers as possible in 10 seconds. Schieber found that the mean search times were longer for the older subjects than for the younger subjects, suggesting that older people have a less efficient search process.

This study examined younger and older people's eye movements in task-directed and task-undirected visual search. For the directed visual search we used Schieber's (1989) CVRT test picture, and examined the search times, number of fixations, and fixation durations as the subjects' gaze traveled from one digit to the next. For the undirected search we used pictures of actual traffic scenes, and examined the number of fixations and fixation durations in pre-determined areas of interest of the visual scene; areas judged to be important for safe driving. This combination of directed and undirected search tasks enabled us to examine more closely age-related differences in visual search. 
Maltz

\section{METHOD}

Subjects: Ten subjects, six females and four males, participated in the experiment. The subjects were evenly divided into two groups: A younger group ranging in age from 20 to 30, with a median age of 26, and an older group ranging in age from 62 to 80 with a median age of 66 . All subjects were licensed drivers with two or more years of driving experience (median driving experience was 8 years for the younger group and 37 years for the older group). All subjects had Snellen acuity of 6/9 (20/30 equivalent) or better with both eyes. None of the subjects wore eyeglasses for the experiment (or to drive), although two of the younger subjects wore contact lenses.

Stimulus Photos and Apparatus: Photographs of actual traffic scenes were shot with a 35mm camera from the driver's seat of an automobile on downtown streets in Beer-Sheva, Israel. Four pictures were chosen for the experiment, based upon clearly visible, safety-related identified areas of interest. These included pedestrians and oncoming, passing, and intersecting vehicles. The pictures chosen were uncluttered, so that the areas of interest could be easily defined. The photographs were digitized via a scanner into bit map images with 256 colors. In addition, two versions of a black and white photograph that was used by Schieber in his Complex Visual Reaction Time Test [1989], one with and one without numeric target overlay, were added to the four, to make a total of six stimulus images for the experiment.

The images were projected by a 386 PC working under Windows 3.1 onto a 14" SVGA color monitor. The monitor was placed on an adjustable table-mounted stand. A combination head and chin rest was mounted on a table facing the monitor. The subject's chair, head rest, and monitor stand were all adjusted so that irrespective of subject height or facial dimensions, the center of the displayed image was aligned at the subject's eye level at a distance of $60 \mathrm{~cm}$ from the subject's eyes. This provided an optical image subtending \pm 12 degrees horizontally and \pm 9 degrees vertically. 
Maltz

The ASL 210 eye tracking system based on electro-optical sensing was used to monitor subject eye movements. The Spectra-Sensors, the apparatus used to collect the eye movement data, contained a sensor unit for each eye consisting of an infrared LED flanked by two phototransistors to measure the corneal reflected light. The phototransistors were positioned to track vertical eye movements of the left eye, and horizontal eye movements of the right eye. The maximum horizontal and vertical movement of the eye able to be tracked by the device was $\pm 15^{\circ}$ from the center. Once calibrated, the system provided an accuracy level of $\pm 1^{\circ}$ horizontally and $\pm 2^{\circ}$ vertically.

Procedure: Each subject was administered a test of binocular visual acuity using a Snellen chart. The subject was presented with a sample image (the black and white photograph without numerical overlay) for familiarization. Subsequently, six images were presented: the aforementioned four traffic scenes taken in downtown BeerSheva and twice the numerical overlay image. The order of the four traffic scene photos was counterbalanced across subjects. The numerical overlay image was always shown second and fifth in the series. Then, the subject was given the CVRT test.

For the numerical overlay image, the subject was instructed to first focus on the number "one" (see Figure 1) and then to locate the numbers in ascending order, until the number "fourteen" was reached. Each subject was allowed the amount of time required to successfully locate all of the numbers in correct order. As each number was found, the subject pressed a button. For the traffic images, the subjects were instructed as follows: "Look at each picture as if you are the driver. You may assume that the car is moving. Pay particular attention to anything that you think is relevant to your continued safe driving of this car." Ten seconds scanning time was allocated per image.

The resulting eye movements were recorded at a sample rate of $60 \mathrm{~Hz}$ with a 486 PC. We used the eye tracker's companion data analysis software, EYENAL, Ver. 
Maltz

2.3. Fixations were defined as mean $x y$ coordinates of eye position lasting over a minimum of 100 milliseconds during which eye position remained within $1^{\circ}$.

\section{RESULTS and DISCUSSION}

Numerical Overlay Pictures:

Search Times: The mean search time to complete the task for the older subjects was significantly higher and more variable than that for the younger subjects. The older group showed a 27.0 seconds mean search time with a standard deviation of 13.1, whereas the younger group's mean search time was 16.7 seconds with a standard deviation of $5.0[t=2.33 ; p=.01]$. However, the distribution of search times for the younger and the older subjects overlapped; a few of the older subjects did rather well in their overall search for the numbers, even comparing favorably with some of the younger subjects' search times.

Insert Figure 1 about here

To gain further insight into the search and scan pattern of the older population, search times between consecutive numbers were analyzed. The mean search time for older subjects was 2.1 seconds compared with 1.3 seconds for younger subjects. Figure 2 shows a histogram of the younger and older populations' search times. As can be seen in the figure, again the older subjects' search times were characterized by high variability (standard deviation of 1.7 compared to .5 for the younger subjects). The medians, however, were similar (1.5 for the older; 1.3 for the younger subjects). To test for statistical significance, a three-way analysis of variance of the search times was performed (age group (2) x picture presentation (2) $x$ searches within picture (13)). Picture presentation and searches were treated as repeated measures. Age was not found to be significant $[F(1,8)=3.27 ; p=.11]$. There were no significant effects or interactions of interest. 
Maltz

Insert Figure 2 about here

Although age was not significant, an examination of the histogram in figure 2 showed that several searches by older subjects were exceptionally long. To ascertain whether the data were unduly influenced by one or two exceptionally slow subjects, or whether each of the older subjects had occasionally long search times for a particular number, we examined the three longest search times for each subject. This technique, known as the application of a non-linear ordered statistics filter has proven to be very useful in target detection tasks, especially to isolate exceptional data for analysis. When averages are used for analysis, outlying data points, while affecting the mean, are lost to the analysis as important effects in and of themselves (see Bar-Tal and Rotman, 1996; Coyle et al., 1989). As can be seen in the histogram in Figure 3, the longest search times of the older subjects were significantly longer than those of the younger group (means were 5.1 and 2.1 with standard deviations of 2.5 and 0.6 respectively, $\mathrm{t}(29)=4.7, \mathrm{p}<.001)$. Thus, it appears that the older subjects were subject to occasional poor performance, while the younger subjects were not.

Insert Figure 3 about here

Fixations: The average fixation duration was 0.27 seconds. A 2 (age) X 2 (picture presentation) ANOVA conducted on the mean fixation durations revealed no significant main effects or interaction. However, an ANOVA of the number of fixations showed significantly more fixations for the older group; with a mean of 87 fixations for overall picture search (of all 14 numbers) compared to 55.7 fixations for the younger group $(F(1,8)=16.85 ; p=.003)$. Thus, the longer total search time for the older subjects was due to an increase in the number of fixations rather than an increase in their duration. This could either be due to a poor visual search process or to a failure to extract the maximum amount of information from each foveal fixation. 
Maltz

Saccades: Mean saccade amplitude (average angular distance jumped between fixations) was similar for the two groups (6.7 degrees for the younger and 6.1 degrees for the older group). A 2 (age) $\times 2$ (picture presentation) ANOVA conducted on the mean saccade amplitude yielded only a significant main effect of the picture presentation: for both groups there was a significant drop in saccade amplitude from the first presentation (mean of 6.7 degrees) to the second one (mean of 6.1 degrees $),(F(1,8)=11.04 ; p=.01)$.

Since no discernible differences in the average saccade amplitude between older and younger subjects were found in the data obtained during their search for all of the numbers in the image, we applied the aforementioned ordered statistic filter to the between-number searches. This was done by examining each subject's average saccadic amplitude during the longest between-number searches within the two pictures. We found that search duration correlated negatively with the mean saccade amplitude $(r=-.45 ; p=.01)$, thus showing that when subjects were having a difficult (i.e. longer) search, their saccade amplitudes tended to be shorter. The correlation between search time and saccade amplitude is shown in Figure 4. [Note that if the single outlying data point - where one of the three long searches of one subject was accompanied by a long saccade - is removed from the calculations, the negative correlation coefficient increases from $r=-.45$ to $r=-.69$.]

Insert Figure 4 about here

CVRT: In addition to measuring the eye movements of our subjects, we separately administered Schieber's CVRT test to each subject and correlated the subjects' test scores with their results from the search of the numerical overlay image. The CVRT scores indicate the number reached (out of 14 ) in a 10-second test. The number of fixations correlated well with the subject's test scores $[r=-.73 ; p=.02]$, and there was a lower correlation between saccadic amplitude and CVRT, [r=.62; $p=.06]$. There was also a negative correlation between CVRT test score and search time, 
Maltz

but its statistical significance was marginal (with $n=10, r=-.45 ; p=0.1$ ). Mean fixation duration did not correlate with CVRT score $(r=.06)$.

Comparing Visual Search of the Numerical Overlay and Traffic Scene Images:

To see if saccadic amplitude was a reliable measure of individual differences, we took the mean saccadic amplitudes for all subjects in their viewing of the numerical overlay picture, and correlated them with the subjects' mean saccadic amplitudes when viewing a traffic scene picture. Although the actual mean saccadic values for the two different types of images were different (6.6 degrees for the numerical overlay picture versus 4.8 degrees for the traffic scene picture), there was a high correlation between the sets of images $(r=0.79 ; p=.01)$, showing that saccadic amplitude is also a consistent (though not necessarily constant) feature of a person's visual search behavior. Previous research has shown that contextual variables - such as the density of information on the image - have a strong influence on saccadic extent [Rayner and Pollatsek, 1992]

\section{$\underline{\text { Traffic Scene Images }}$}

We divided the four traffic scene images into areas of interest (AOI) appropriate for driver attention. AOls were defined as areas containing information relevant to immediate safety of the driver, such as mirrors and other cars/pedestrians who may impede or cross the path of travel. Figure 5 shows one of the images with its AOls delineated.

Insert Figure 5 about here

After defining the AOls, we checked how the subjects divided their visual search between the AOls and the areas outside the AOls, by comparing dwell times; the total time - of all saccades and fixations - spent in an area. Across images, mean dwell percent-of-time outside the AOls was 20-34 (with standard deviations of 12-14 $\%)$. There was no significant effect of age $[F(1,6)=2.39 ; p=.17]$. 
Maltz

For a more detailed analysis, we examined the visual search of each image by calculating the percentages of dwell time, in each of the AOls for that image. We then tested for differences between the younger and older groups. Two way ANOVAs of the effects of Age X AOls on percent dwell time were conducted on the four images. While there were no differences in total dwell time found between the groups in any of the images based on age alone, for two of the images there were significant interactions between age group and $A O I$ : $F(3,18)=10.5 ; p<.001$ for image 3 and $F(2,16)=4.01 ; p=.04$ for image 4 . In both of these images the older subjects tended to ignore some AOls while focusing inordinately long on a subset of the AOls.

To quantify this effect and provide a possible explanation for it, we defined a measure - the 'Concentration Ratio' - which is the degree that certain areas are fixated 'more than expected'. The 'expected' level was the percent of the area of the AOls relative to the total image. As a baseline, we assumed that totally random scanning would exhibit fractional dwell times equal to the fraction of the screen that the AOI occupies. As a subject concentrated on one area more than on others, his/her fraction on that particular AOI would go up and the others would fall. By calculating and summing over all the AOls the absolute value of the difference between the actual percent dwell times on the AOls and the percent of each area out of the total scene, we could determine if older people really concentrate their fixations on a more limited number of AOls. If we define the percentage area of the $i^{\text {th }} A O I$ to be $A_{i}$ and the actual percentage of fixations in the $i^{\text {th }} A O I$ to be $F_{i}$, then our Concentration Ratio is: $\sum_{i}\left|F_{i}-A_{i}\right|$. Note that the introduction of the absolute value guarantees that a subject who looks more evenly over the AOls (proportionally to their size) will have the lowest values. Figure 6 illustrates the results of this test. 
Maltz

As hypothesized, the older subjects, did show increased concentration in a smaller number of AOls. The mean Concentration Ratio of the older group (62.2) was significantly higher than that of the younger group $(36.7),[F(1,6)=18.5 ; p=.005]$. There was also a significant interaction between image and age $[F(3,18)=6.13$; $p=.005]$, reflecting a difference in the concentration ratio in three out of the four images only.

Two possible visual search models could account for the differences between the older and younger subjects. One possibility is that the older subjects limit the number of AOls that they scan, and dwell on them for longer periods of time. According to this model, the average scan of an $\mathrm{AOI}$ is uninterrupted by eye movements to other AOls and is longer for the older than for the younger subjects. A second possibility is that older subjects' fixations jump from one AOI to the next, and the limited number of AOls they fixate reflects saccadic movements to AOIs that have already been scanned.

To evaluate the two models, we examined the ratio of simple to conditional probabilities of fixating on an $\mathrm{AOI}$. The percent dwell time in each $\mathrm{AOI}$ was used as the "simple" probability of a subject's fixation on a particular AOI. In addition, we calculated the conditional probability of saccadic movements from one AOI to another. This ratio of conditional probability to the simple probability, $P_{x \mid y}(x \mid y) / P_{x}$ $(x)$, is a 'stability ratio' for $y=x$. If the ratio is close to one, then the subject's fixation pattern is fairly random or uniform across the whole image, since his/her fixation in a particular AOI is independent of the previous fixation. As the ratio gets higher, the visual search becomes "stable" (i.e., once scanning an AOI, the subject tends to continue scanning that $\mathrm{AOI}$ ). In this way, we hoped to tell if the older subjects were limiting the number of AOls on which they fixated because they were more stable and, hence, spent more time in fewer AOls, or if they were visually jumping from one $\mathrm{AOI}$ to the next and simply returning to AOls already fixated without fixating all AOls. 
Maltz

For this analysis, we focused on the AOI with the highest dwell time in each image, so that a statistically significant number of fixations would be available for statistical analysis. To avoid biasing the analysis, we chose the most fixated $\mathrm{AOI}$ for each image by selecting the $\mathrm{AOI}$ in which the most subjects across both age groups had the highest dwell times. Since there was a clear preference shown in each image (even the younger group had favored areas; they just scanned relatively more evenly than the older group) there was no need to make subjective judgments. For example, for the image in Figure 5, the most fixated AOI was the car on the right.

The results of the two-way ANOVA of age $x$ image revealed a clear-cut effect of age: the younger subjects were nearly twice as stable as the older subjects, with a mean stability ratio of 2.39 compared to the older group's mean stability ratio of 1.27 $[F(1,6)=13.02 ; p=.01]$. This age effect was obtained for each of the images, as can be seen in Figure 7. The main effect of image and the interaction between image and age were not significant $[F(3,18)=.69 ; p=.57$, and $F(3,18)=.39 ; p=.76$, respectively]. Hence, not only was age alone significant, but the direction of the difference in the means is consistent with the previous analysis of fixation durations and number of fixations. The older subjects' tendency to focus on fewer AOls was apparently not due to a slower rate of information extraction or intense interest in those areas. Instead, as proposed above by the second model, it was due to a scattering of fixations (and probably attention) that caused the subjects to repeatedly return to the few AOls they had already scanned.

Insert Figure 7 About Here

\section{CONCLUSIONS}

The results of both parts of this study, the directed visual search task (with the numerical overlay) and the undirected visual search (with the actual traffic scenes), 
Maltz

point to variability in performance of visual-related tasks by older persons. The variability that we found in our older subject group is consistent with previous findings of increased individual differences among the older population in basic psychomotor functions as well as in driving related skills [e.g. Moschner and Baloh, 1994; Ball et al., 1993].

The visual search task demonstrated that older persons need longer visual search times than younger persons to extract the same information. Furthermore, the nature of the search process provides insight into why older people require more time to perform the CVRT task. Older people's visual search behavior is characterized by (a) large variability in the data from the older group which was not present in the younger group, and (b) occasional lapses which resulted in searching difficulty. During the lapses in which the older subjects had difficulty searching, their eye movements were characterized by shorter saccades and increased numbers of fixations. This could indicate a poor visual search process with ineffective use of the peripheral field, or a failure to extract maximum amounts of information from areas already fixated. It is possible that these lapses that we observed among the older subjects reflect the "visual inattention" that Ball et al. [1993] cite as contributing to the crash frequency of older drivers more than their sensory visual limitations. Occasional lapses could very well be seen as driver inattention, which also occurs sporadically.

The Concentration Ratio values obtained from the visual search of real traffic scenes suggest that the older subjects focus on a smaller subset of the areas of interest within the images than younger subjects who distribute their attention more evenly across different parts of the scene. Here too, older subjects exhibited more variability in their behavior, with some of their scans as even as those of the younger subjects' scans. The older subjects were consistent in their lack of stability in focusing on the AOIs, as shown by our Stability Ratio. The combination of the older subjects' tendency to scan fewer of the AOls with their greater tendency to dart from one $\mathrm{AOI}$ to another, is also an indication of inefficiency in both the visual search and information extraction. 
Maltz

Finally, it is noteworthy that the saccadic movements exhibited by both age groups during both tasks were consistent within subject. This would indicate that people tend to have the same general type of saccadic movement (i.e., short or long) for different types of tasks and that saccadic eye movements may be a stable indication of individual differences. Further study of eye movements is warranted to explore this further.

In summary, the results of this study show that older persons suffer occasional lapses during visual search and that their visual behavior in general is more variable than that of younger people. This behavior might reflect age-related difficulties in sustained attention. Whether these occasional lapses are serious enough to warrant labeling the older population as poorer drivers remains questionable, given their overall performance and tendency to compensate by self-restrictions to drive less, and to drive in less demanding situations (e.g., Campbell, et al., 1993; Zur and Shinar, 1998). Furthermore, the variability in the performance of the older subjects shown here and in other studies highlights the inadvisability of characterizing all older persons in terms of stereotypic labels related to driving and other visuallydependent tasks.

\section{ACKNOWLEDGEMENTS}

We are thankful to Frank Schieber for providing us with the CVRT stimuli, to Joseph $\mathrm{H}$. Goldberg, for his comments on an early draft of this paper, and to Stanley R. Rotman for his contributions to the data analysis.

\section{REFERENCES}

Ball, K., Beard, B., Roenker, D., Miller, R., and Griggs, D. (1988). Age and visual search: Expanding the useful field of view. Journal of the Optical Society of America, $\underline{5}, 2210-2219$.

Ball, K., and Owsley, C. (1991). Identifying correlates of accident involvement for the older driver. Human Factors, $\underline{33}$, 583-595. 
Maltz

Ball, K., Owsley, C., Sloane, M.E., Roenker, D.L., and Bruni, J.R. (1993). Visual attention problems as a predictor of vehicle crashes in older drivers. Investigative Ophthalmology and Vision Sciences, 34(11), 3110 -3123.

Ball, K., and Rebok, G. (1994). Evaluating the driving ability of older adults. Journal of Applied Gerontology, 13(1), 20-38.

Bar-Tal, M. and Rotman, S.R. (1996). Performance measurement in point source target detection. Infrared Physics and Technology, 37, 233-238.

Bono, F., Oliveri, R.L., Zappia, M., Aguglia, U., Puccio, G., and Quattrone, A. (1996). Computerized analysis of eye movements as a function of age. Archives of Gerontology and Geriatrics, 22(3), 261-269.

Campbell, M.K., Bush, T.L., and Hale, W.E. (1993). Medical conditions associated with driving cessation in community-dwelling, ambulatory elders. Journal of Gerontology, 48 (4), S230-S234.

Coyle, E.J., Lin, J., and Gabbouj, M. (1989). Optimal stack filtering and the estimation and structural approaches to image processing. IEEE Trans. on Acoustics, Speech, and Signal Processing, 37(12), 2037-2066.

Kanayama, R., Nakamura, T., Sano, R., Ohki, M., Okuyama, T., Kimura, Y., and Koike, Y. (1994). Effect of aging on smooth-pursuit eye-movement. Acta OtoLaryngologica S511, 131-134.

Kline, D.W. (1994). Optimizing the visibility of displays for older observers. Experimental Aging Research 20(1), 11-23.

McMahan, P.B. (1973). An Analysis of Factors That Contribute to the Performance of a Driver Eye Movement Recording System. MS thesis, Ohio State University, Dept. of Industrial and Systems Engineering.

Moschner, C. and Baloh, R.W. (1994). Age-related changes in visual tracking. Journals of Gerontology 49(5), M235-M238. 
Maltz

Olincy, A., Ross, R.G., Young, DA, and Freedman, R. (1997). Age diminishes performance on an antisaccade eye-movement task. Neurobiology of Aging, 18(5), 483-489.

Owsley, C., Ball, K., Sloane, M.E., Roenker, D.L., and Bruni, J. R. (1991).

Visual/cognitive correlates of vehicle accidents in older drivers. Psychology and Aging, $\underline{6}$, 403-415.

Rackoff, N.J. (1975). An investigation of age-related changes in drivers visual search patterns and driving performance and the relation to tests of basic function capacities. In Proceedings of the Human Factors Society 19th Annual Meeting (pp. 285-288).

Rayner, K., and Pollatsek, A. (1992). Eye movements and scene perception. Canadian J Psychology, 46(3), 342-376.

Rockwell, T.H. (1972). Skills, judgment and information acquisition in driving. In T.W. Forbes (Ed.), Human Factors in Highway Traffic Safety Research (pp. 133-164). New York: Wiley-Interscience.

Schieber, F. (1989). The complex visual reaction time test (Tech. Report). Oakland University, Dept. of Psychology.

Serafin, C. (1994). Driver eye fixations on rural roads: insight into safe driving behavior (Tech. Report UMTRI-94-21). The University of Michigan Transportation Research Institute.

Shinar, D. (1977). Driver visual limitations: diagnosis and treatment. Bloomington: Indiana University, Institute for Research in Public Safety.

Shinar, D. (1978). Field dependence and driver visual search behavior. Factors, 20(5), 553-559.

Shinar, D. and Schieber, F. (1991). Visual requirements for safety and mobility of

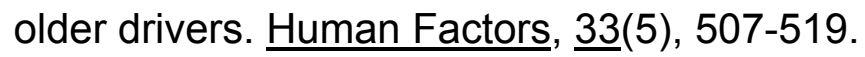


Maltz

Staplin, L.K., Breton, M.E., Haimo, S.F., Farber, E.I., and Byrnes, A.M. (1987). Agerelated diminished capabilities and driver performance. (Task A Report, USDOT/FHWA Contract No. DTFH61-86-C-00044). Malvern, PA: KETRON, Inc.

Szlyk, J.P., Seiple, W., and Viana, M., (1995(a)). Relative effects of age and compromised vision on driving performance. Human Factors, 37(2), 430-436.

Szlyk, J.P., Pizzimenti, C.E., Fishman, G.A., Kelsch, R., Wetzel, L.C., and Kagan, S. (1995(b)). A comparison of driving in older subjects with and without agerelated macular degeneration. Archives of Ophthalmology, 113, 1033-1040.

Whalen, J.T., Rockwell, T.H. and Mourant, R.R. (1968). a pilot study of drivers' eye movements. (Interim Report No. 277B-1). Engineering Experiment Station, The Ohio State University.

Whiteside, J.A. (1974). Eye movements of children, adults, and elderly persons

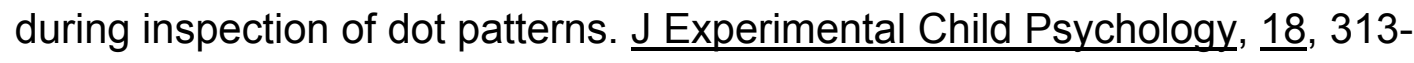
332.

Zur, A. and Shinar, D. (1998). Older people's driving habits, visual abilities, and subjective assessment of daily visual functioning. Work, in press. 
Maltz

MASHA MALTZ received the MBA degree in Computer Methodology from Bernard Baruch College, CUNY in 1982. She is currently a doctoral student in the Department of Industrial Engineering and Management at Ben-Gurion University of the Negev conducting research towards her Ph.D. degree. Her present research interests include cueing systems and eye movements, and their relation to traffic safety and target acquisition.

DAVID SHINAR received his Ph.D in human performance and human factors from the Ohio State University in 1973. He is a professor of Ergonomics in the Department of Industrial Engineering and Management at Ben-Gurion University of the Negev. His research interests include applied vision and information processing. driver behavior, and traffic safety. 


\section{List of Figures}

Figure 1. The numerical overlay image used in the CVRT.

Figure 2. The distribution of all search times between numbers as a function of age.

Figure 3. The distribution of the longest search times as a function of age.

Figure 4. The correlation between search time and saccade amplitude (after application of ordered statistics filter, $n=30$ ).

Figure 5. Traffic scene image 3 with four areas of interest (left to right: oncoming traffic, cars ahead, car right, mirror).

Figure 6 . The relationship between the percent time spent in each $\mathrm{AOI}$ and the fraction of its size out of the total image (for image 3 ).

Figure 7. Stability Ratio of older and younger subjects in each of the four images. 

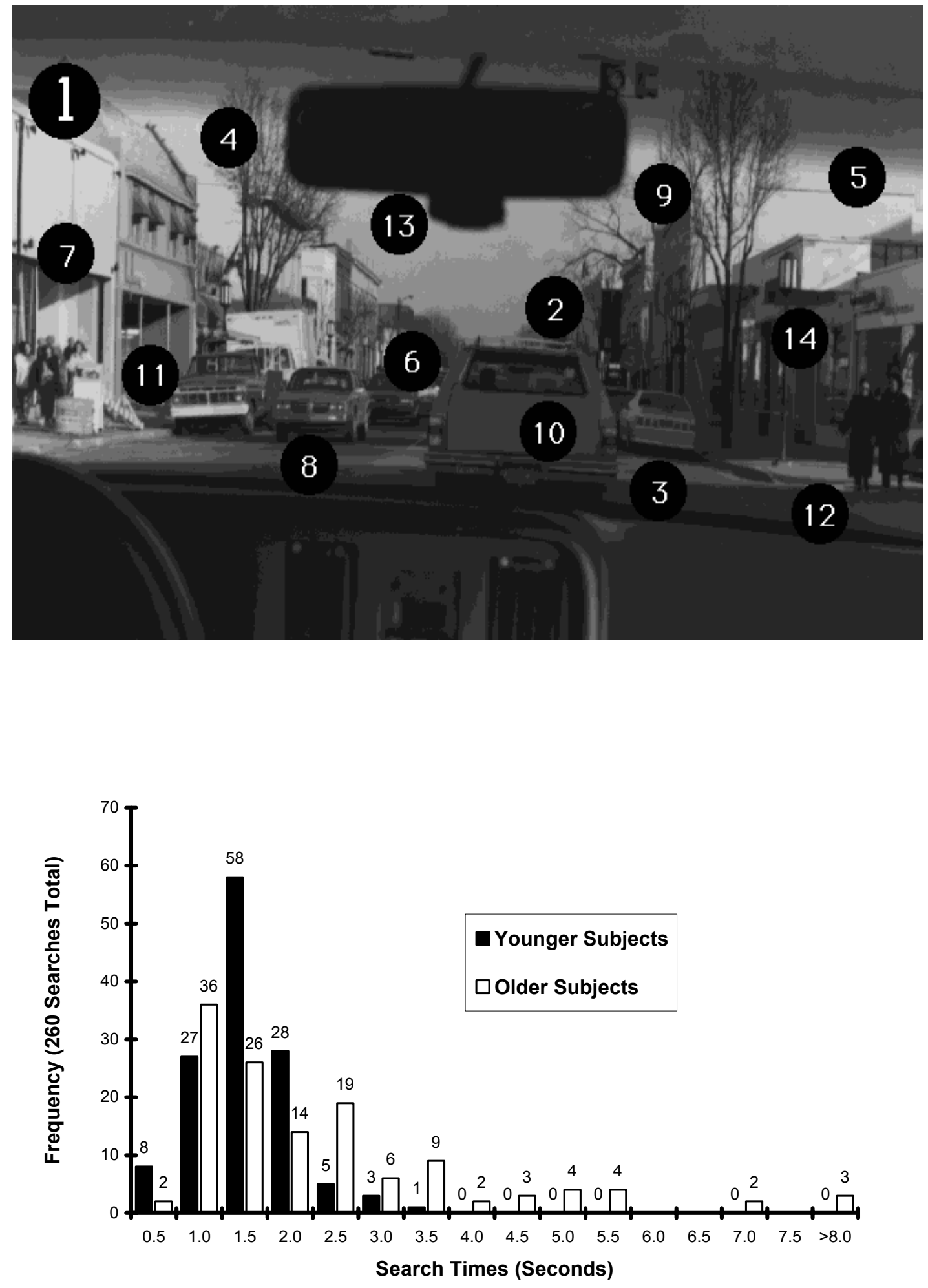

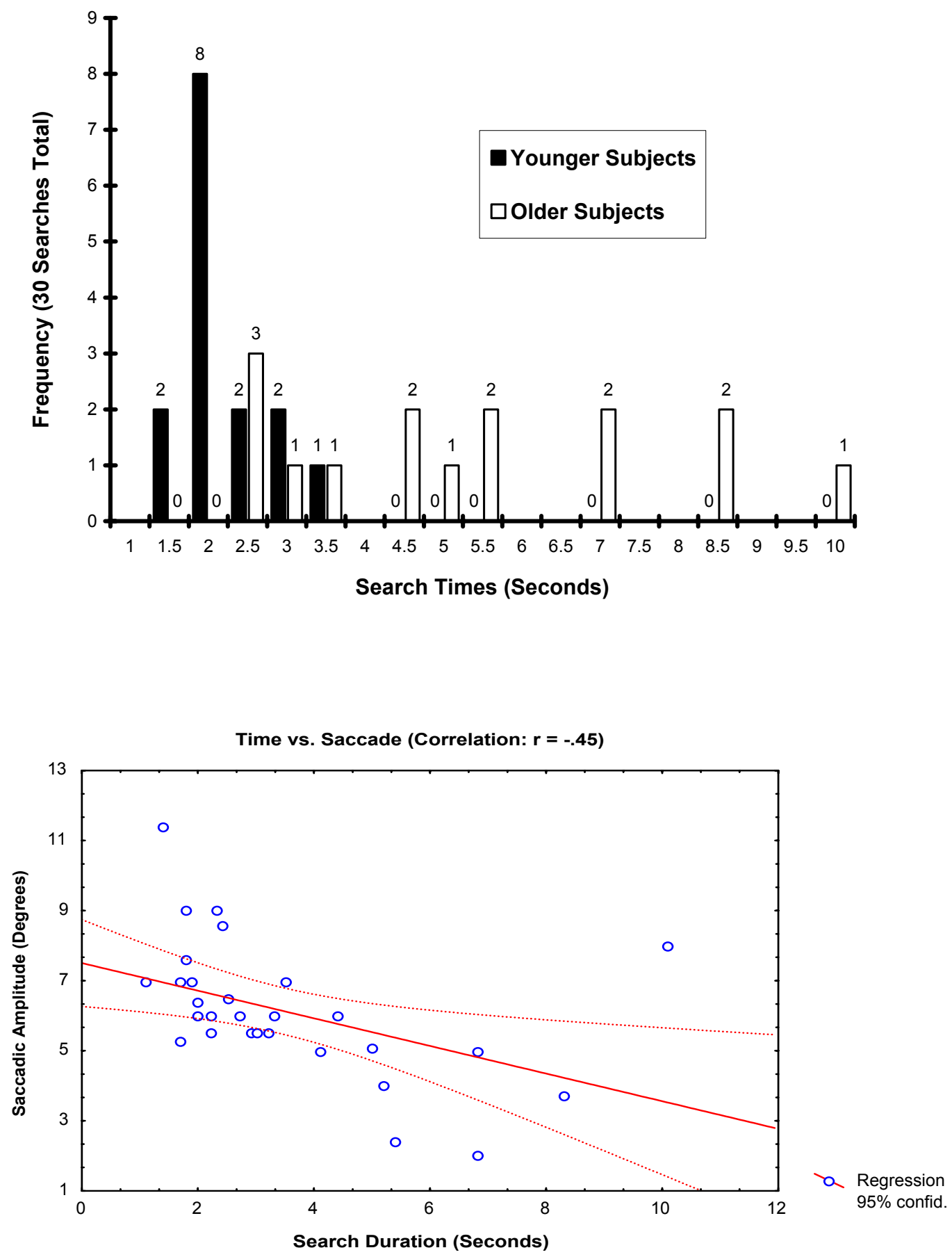

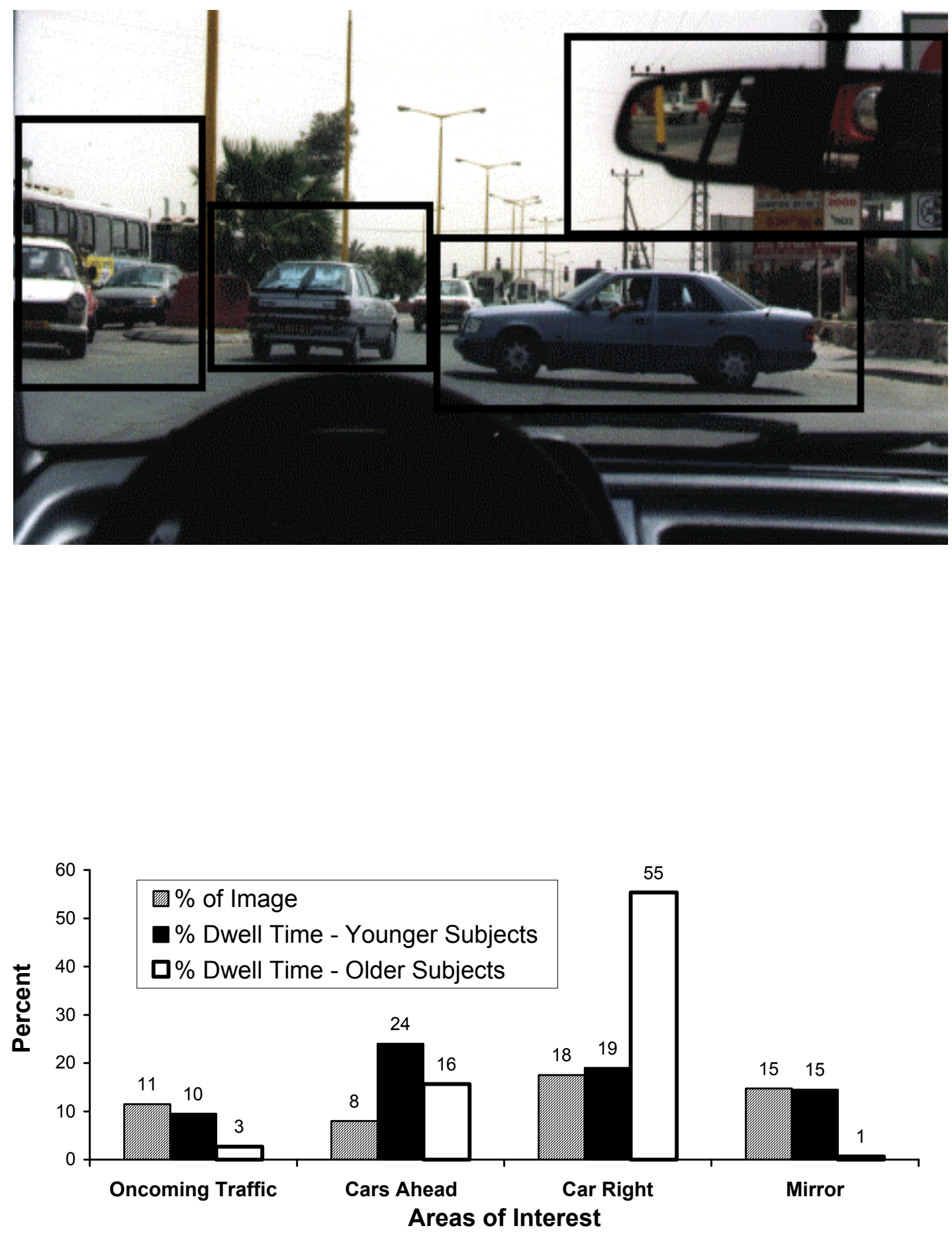


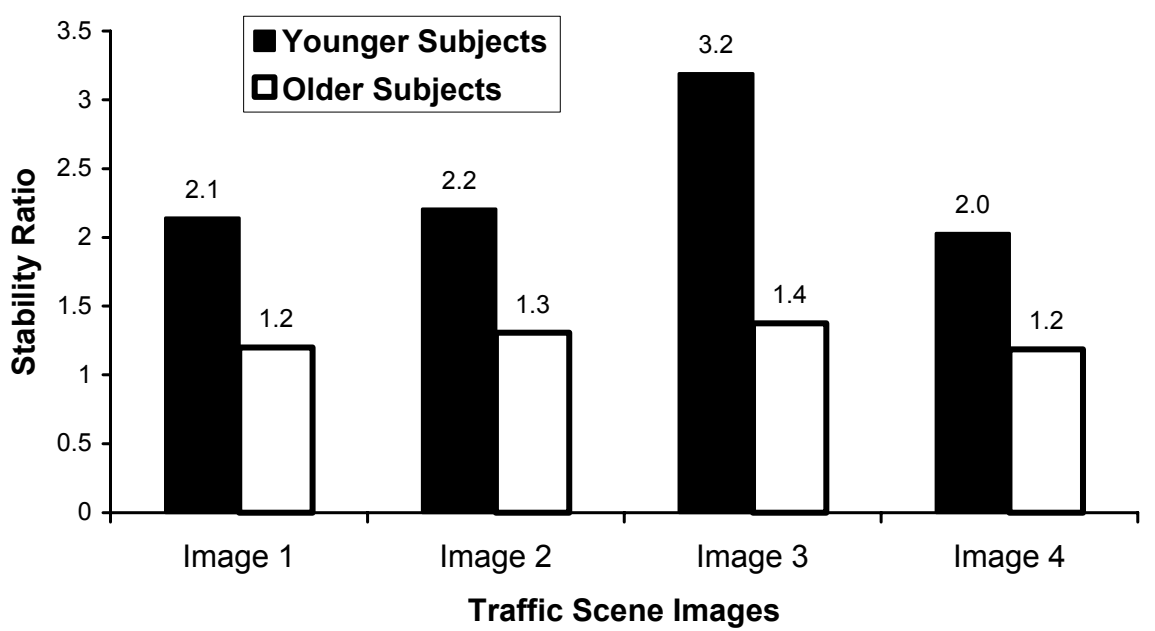

